\title{
Methods of health risk assessment for human exposure to high frequency electromagnetic fields
}

\author{
D. Poljak ${ }^{1}$, M. Cvetković ${ }^{1} \&$ H. Dodig ${ }^{2}$ \\ ${ }^{1}$ University of Split, Croatia \\ ${ }^{2}$ Marine Electronics Center, Croatia
}

\begin{abstract}
The paper reviews some topics in human exposure to high frequency (HF) electromagnetic fields based on certain integral equation formulations and related numerical solution procedures. Illustrative computational examples are related to the human eye and the human brain exposed to HF radiation. The obtained numerical results for specific absorption rate (SAR) are compared against exposure limits proposed by ICNIRP (International Commission on Non Ionizing Radiation Protection).
\end{abstract}

Keywords: human exposure, high frequency radiation, specific absorption rate, integral equation formulation, numerical solution procedures.

\section{Introduction}

The tremendous growth of wireless communication systems in modern society has increased the public concern regarding possible adverse health effects due to exposure to high frequency (HF) radiation. The HF exposure assessment is based on the calculation of SAR distribution.

A comprehensive review on this hot issue could be found in many papers, e.g. [1-3]. As measurement of induced fields in the body is not possible human exposure assessment is carried out by using appropriate computational models [2-5]. Theoretical models are being used to simulate various exposure scenarios, and thereby establish safety guidelines and exposure limits for humans [6, 7]. Computational models can be classified as either realistic models of the body (or particular organs) mostly based on Magnetic Resonance Imaging (MRI) [2, 3, 8- 
10], or simplified models, computationally much less demanding but failing to provide accurate results in most of the exposure scenarios.

This paper reviews the use of integral equation approaches in bioelectromagnetics developed by the authors.

Related illustrative computational examples presented in the paper are related to the human eye and the human brain exposed to HF radiation $[11,12]$. The eye exposure is based on the hybrid boundary element/finite element method (BEM/FEM) of solution of the Helmholtz equation. The brain exposure is analyzed by solving the set of coupled surface integral equations (SIEs) via the Method of Moments (MoM). The obtained maximal values of SAR are compared to the exposure limits proposed by ICNIRP.

\section{HF dosimetry basics}

The principal task of HF dosimetry is related to the assessment of thermal effects, i.e. to determine the level and distribution of the electromagnetic energy absorbed by the body. The main dosimetric quantity for quantifying the influence of $\mathrm{HF}$ fields is the specific absorption rate (SAR) and related temperature increase.

Due to the mathematical complexity of the problem most of the early stage researchers investigated simple models such as plane slab, cylinders, homogeneous and layered spheres and prolate spheroids [13] while recent anatomically based computational models comprising of cubical cells are dominantly related to the application of the Finite Difference Time Domain (FDTD) methods. The Finite Element Method (FEM) and Boundary Element Method (BEM) are generally used to a somewhat lesser extent [13-15].

The specific absorption rate $(S A R)$ represents a fundamental quantity in HF dosimetry. $S A R$ is defined in terms of the rate of energy $W$ absorbed by, or dissipated in the unit body mass $m$ :

$$
S A R=\frac{d P}{d m}=\frac{d}{d m} \frac{d W}{d t}=C \frac{d T}{d t}
$$

where $P$ stands for dissipated power, $C$ is the specific heat capacity of tissue, $T$ is the temperature and $t$ denotes time.

Also, $S A R$ is proportional to the square of the internal electric field:

$$
S A R=\frac{d P}{d m}=\frac{d P}{\rho d V}=\frac{\sigma}{2 \rho}|E|^{2}=\frac{\sigma}{\rho}\left|E_{r m s}\right|^{2}
$$

where $E$ and $E_{\mathrm{rms}}$ is the peak and root-mean-square value of the electric field, respectively, $\rho$ is the tissue density and $\sigma$ is the tissue conductivity.

$S A R$ distribution generally depends on the incident field parameters, the characteristics of the exposed body, ground and reflector effects, respectively. $S A R$ of the biological body reaches maximal values when the electric field is oriented parallel to the long body axis. 


\section{The eye exposure to $\mathrm{HF}$ radiation}

The human eye exposed to plane wave represents an electromagnetic scattering from lossy dielectric object. The eye model is based on the Stratton-Chu formulation and Helmholtz equation. The hybrid BEM/FEM approach with edge elements is applied to the assessment of related internal fields.

\subsection{Formulation}

A plane wave incident on the corneal part of the eye represents an unbounded scattering problem (as shown in Fig. 1).

According to Stratton-Chu formulation, the time-harmonic electric field occurring at the exterior of the scattering problem is expressed by the following boundary integral equation [11]:

$$
\alpha \vec{E}=\vec{E}_{i}+\oint_{\partial V^{\prime}} \vec{n}^{\prime} \times\left(\nabla^{\prime} \times \vec{E}\right) G d S^{\prime}+\oint_{\partial V^{\prime}}\left[\left(\vec{n}^{\prime} \times \vec{E}\right) \times \nabla^{\prime} G+\left(\vec{n}^{\prime} \cdot \vec{E}\right) G\right] d S^{\prime}
$$

where $\vec{E}_{i}$ is the incident field, $\vec{n}^{\prime}$ is an outer normal to surface $\partial V^{\prime}$ bounding the volume $V$ and $\alpha$ is the solid angle subtended at an observation point.

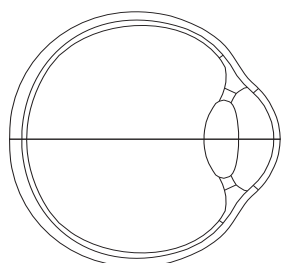

(a)

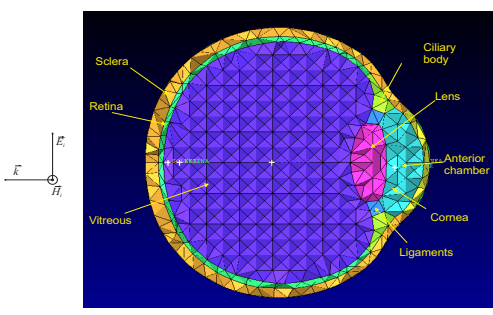

(b)

Figure 1: (a) Model of the eye exposed to plane wave; (b) meshing detail, an eye is modeled with 36027 tetrahedral elements.

Furthermore, $G$ represents the fundamental solution of the Helmholtz equation:

$$
\nabla^{2} G+k^{2} G=-\delta\left(\vec{r}-\vec{r}^{\prime}\right)
$$

The interior of the eye containing inhomogeneous regions is represented by equation [11]:

$$
\nabla \times\left(\frac{1}{k_{B}} \nabla \times \vec{E}\right)-k_{A} \vec{E}=0
$$

where subscripts A and B denote the exterior and interior region, respectively. 


\subsection{Boundary element solution}

The boundary integral equation (3) is handled via the boundary element method (BEM) [3], while the partial differential equation (5) is solved by means of finite element method (FEM).

Namely, applying the weighted residual approach to equation (5) and introducing the vector test functions $\vec{W}_{i}$ the following integral representation is obtained $[3,11]$ :

$$
\int_{V^{\prime}}\left[\nabla \times\left(\frac{1}{k_{B}} \nabla \times \vec{E}\right)-k_{A} \vec{E}\right] \cdot \vec{W}_{i} d V^{\prime}=0
$$

Once the electric field $\vec{E}$ is determined, $S A R$ can be computed from (2).

\subsection{Numerical results}

Fig. 2 shows the numerical results for the SAR distribution within the eye obtained via the hybrid BEM/FEM procedure due to the incident plane wave with power density of $10 \mathrm{~W} / \mathrm{m}^{2}$.

The calculated SAR results presented in this work are in a satisfactory agreement with the results reported in [16].

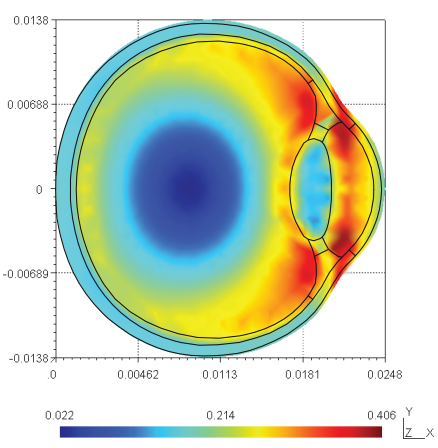

(a)

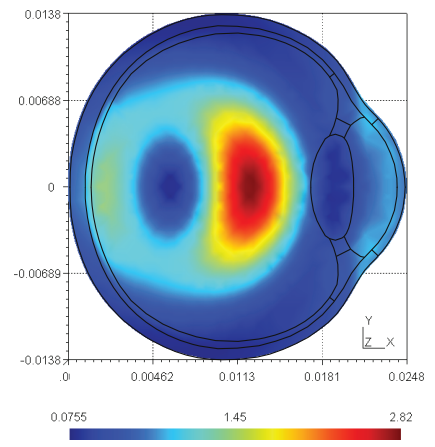

(b)

Figure 2: Computed SAR in the eye induced by plane wave of power density $10 \mathrm{~W} / \mathrm{m}^{2}$ at frequency (a) $1 \mathrm{GHz}$; (b) $2 \mathrm{GHz}$.

The results for SAR at different frequencies, averaged over the whole eye, are given in Table 1.

From the presented numerical results it can be seen that the hot spots formation occurs below the frequencies of $6 \mathrm{GHz}$, while at higher frequencies the electromagnetic energy is focused at the eye surface. Also, the whole eye averaged SAR values stay below the ICNIRP exposure limits [6]. 
Table 1: The whole eye averaged $S A R$ at $f=1 \mathrm{GHz}, 2 \mathrm{GHz}, 4 \mathrm{GHz}$ and $6 \mathrm{GHz}$.

\begin{tabular}{c|c}
\hline $\begin{array}{c}\text { Frequency } \\
{[\mathrm{GHz}]}\end{array}$ & $\begin{array}{c}\text { SAR } \\
{[\mathrm{W} / \mathrm{kg}]}\end{array}$ \\
\hline 1 & 0.3352 \\
2 & 0.6189 \\
4 & 1.2617 \\
6 & 1.0689 \\
\hline
\end{tabular}

\section{The brain exposure to $\mathrm{HF}$ radiation}

The model of the human brain exposed to HF electromagnetic fields is based on the surface integral equation (SIE) approach. The formulation can be derived from the equivalence theorem and by using the appropriate interface conditions for the electric and/or magnetic field, as depicted in Fig. 3.

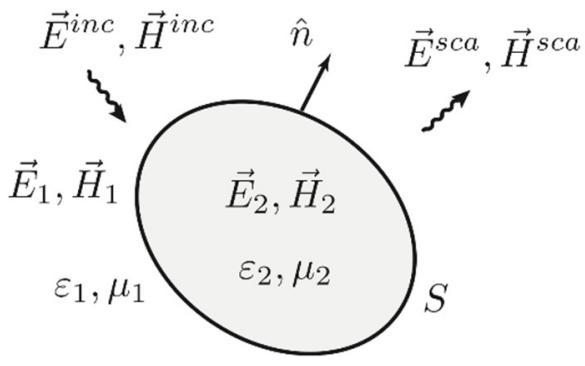

Figure 3: The brain represented by a lossy homogeneous dielectric.

The lossy homogeneous object representing the brain is excited by the incident electromagnetic field ( $\vec{E}^{i n c} ; \vec{H}^{i n c}$ ).

\subsection{Formulation}

Using the equivalence theorem, two equivalent problems can be formulated, in terms of the equivalent electric and magnetic current densities $\vec{J}$ and $\vec{M}$ existing at the surface $S$, one for the region 1 (exterior to dielectric) and other for the region 2 (inside the dielectric) [17-20].

The boundary conditions at the surface $S$ are satisfied by introducing equivalent surface currents $\vec{J}_{2}=-\vec{J}_{1}$ and $\vec{M}_{2}=-\vec{M}_{1}$ at the surface. Following the same procedure for the interior equivalent problem, yields another 
homogeneous domain of ("2;_2). Again, the equivalent surface currents $\vec{J}_{1}$ and $\vec{M}_{1}$ are introduced.

Performing certain mathematical manipulations, the following set of integral equations is obtained [10]:

$$
\begin{aligned}
& j \omega \mu_{n} \iint_{S} \vec{J}\left(\vec{r}^{\prime}\right) G_{n}\left(\vec{r}, \vec{r}^{\prime}\right) \mathrm{d} S^{\prime}- \\
& -\frac{j}{\omega \varepsilon_{n}} \iint_{S} \nabla_{S}^{\prime} \cdot \vec{J}\left(\vec{r}^{\prime}\right) \nabla G_{n}\left(\vec{r}, \vec{r}^{\prime}\right) \mathrm{d} S^{\prime}+ \\
& +\iint_{S} \vec{M}\left(\vec{r}^{\prime}\right) \times \nabla^{\prime} G_{n}\left(\vec{r}, \vec{r}^{\prime}\right) \mathrm{d} S^{\prime}= \begin{cases}\vec{E}^{i n c}, & n=1 \\
0, & n=2\end{cases}
\end{aligned}
$$

where the Greeen's function for the homogeneous medium is given by:

$$
G_{n}\left(\vec{r}, \vec{r}^{\prime}\right)=\frac{e^{-j k_{n} R}}{4 \pi R} ; \quad R=\left|\vec{r}-\vec{r}^{\prime}\right|
$$

and $R$ is the distance from the source to observation point, respectively while $k_{\mathrm{n}}$ denotes the wave number of a medium $n,(n=1 ; 2)$.

\subsection{Solution by the method of moments (MoM)}

The numerical solution of the coupled SIEs is carried out via the method of moments (MoM). A view to the triangular brain model mesh is shown in Fig 4.

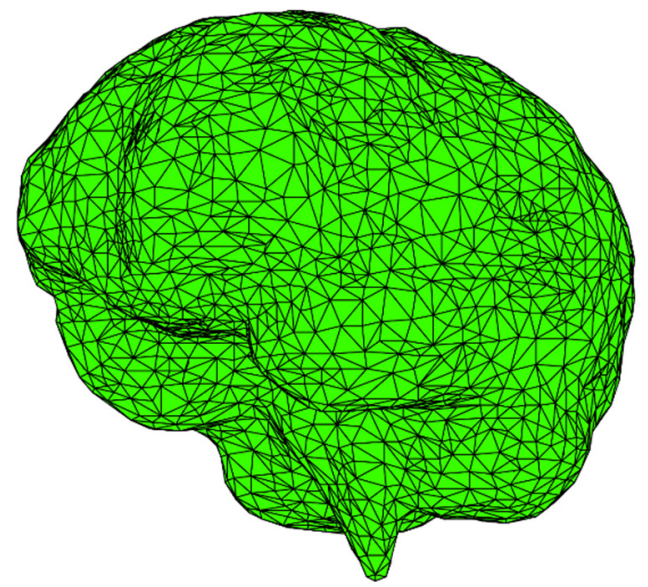

Figure 4: Triangular mesh of the homogeneous brain model. 
First, the equivalent electric and magnetic currents $\vec{J}$ and $\vec{M}$ in (7) are expressed in terms of a linear combination of basis functions $\vec{f}_{n}$ and $\vec{g}_{n}$, respectively.

$$
\begin{gathered}
\vec{J}(\vec{r})=\sum_{n=1}^{N} J_{n} \vec{f}_{n}(\vec{r}) \\
\vec{M}(\vec{r})=\sum_{n=1}^{N} M_{n} \vec{g}_{n}(\vec{r})
\end{gathered}
$$

where $J_{\mathrm{n}}$ and $M_{\mathrm{n}}$ are unknown coefficients, while $N$ is the total number of triangular elements.

Applying the weighted residual approach, i.e. multiplying (7) by the set of a test functions $\vec{f}_{m}$ and integrating over the surface $S$, after performing some mathematical manipulations, it follows:

$$
\begin{gathered}
j \omega \mu_{i} \sum_{n=1}^{N} J_{n} \iint_{S} \vec{f}_{m}(\vec{r}) \cdot \iint_{S^{\prime}} \vec{f}_{n}\left(\vec{r}^{\prime}\right) G_{i} d S^{\prime} d S+ \\
+\frac{j}{\omega \varepsilon_{i}} \sum_{n=1}^{N} J_{n} \iint_{S} \nabla_{S} \cdot \vec{f}_{m}(\vec{r}) \iint_{S^{\prime}} \nabla_{S}^{\prime} \cdot \vec{f}_{n}\left(\vec{r}^{\prime}\right) G_{i} d S^{\prime} d S+ \\
\pm \sum_{n=1}^{N} M_{n} \iint_{S} \vec{f}_{m}(\vec{r}) \cdot\left[\hat{n} \times \vec{g}_{n}\left(\vec{r}^{\prime}\right)\right] d S+ \\
+\sum_{n=1}^{N} M_{n} \iint_{S} \vec{f}_{m}(\vec{r}) \cdot \iint_{S^{\prime}} \vec{g}_{n}\left(\vec{r}^{\prime}\right) \times \nabla^{\prime} G_{i} d S^{\prime} d S= \\
=\left\{\iint_{S} \vec{f}_{m}(\vec{r}) \cdot \vec{E}^{i n c} d S \quad, i=1\right. \\
0 \quad, i=2
\end{gathered}
$$

where subscript $i$ denotes the index of the medium. The details of the procedure could be found elsewhere, e.g. in [10].

\subsection{Numerical results}

Computational examples are related to the brain exposure to plane wave at $f=900$ $\mathrm{MHz}$ and $1800 \mathrm{MHz}$, respectively. Surface of the brain is disretized using 696 triangular elements and 1044 edge-elements while the corresponding brain parameters are listed in Table 2. 
The power density of the incident plane wave is $P=5 \mathrm{~mW} / \mathrm{cm}^{2}$ and is oriented perpendicular to the right side of the brain (positive $\mathrm{x}$ coordinate).

Fig. 5 shows the $S A R$ distribution in the brain at $f=900 \mathrm{MHz}$ for the case of horizontal polarization.

Table 2: $\quad$ Permittivity and conductivity of the brain.

\begin{tabular}{cc|rc}
\hline \multicolumn{4}{c}{ Human brain parameters } \\
\hline \hline \multicolumn{3}{c}{$900 \mathrm{MHz}$} & \multicolumn{3}{c}{$1800 \mathrm{MHz}$} \\
\hline$\varepsilon_{r}$ & $\sigma[\mathrm{S} / \mathrm{m}]$ & $\varepsilon_{r}$ & $\sigma[\mathrm{S} / \mathrm{m}]$ \\
45,8054 & 0,7665 & 43,5449 & 1,15308 \\
\hline \hline
\end{tabular}

\section{SAR $[\mathrm{W} / \mathrm{kg}]$}
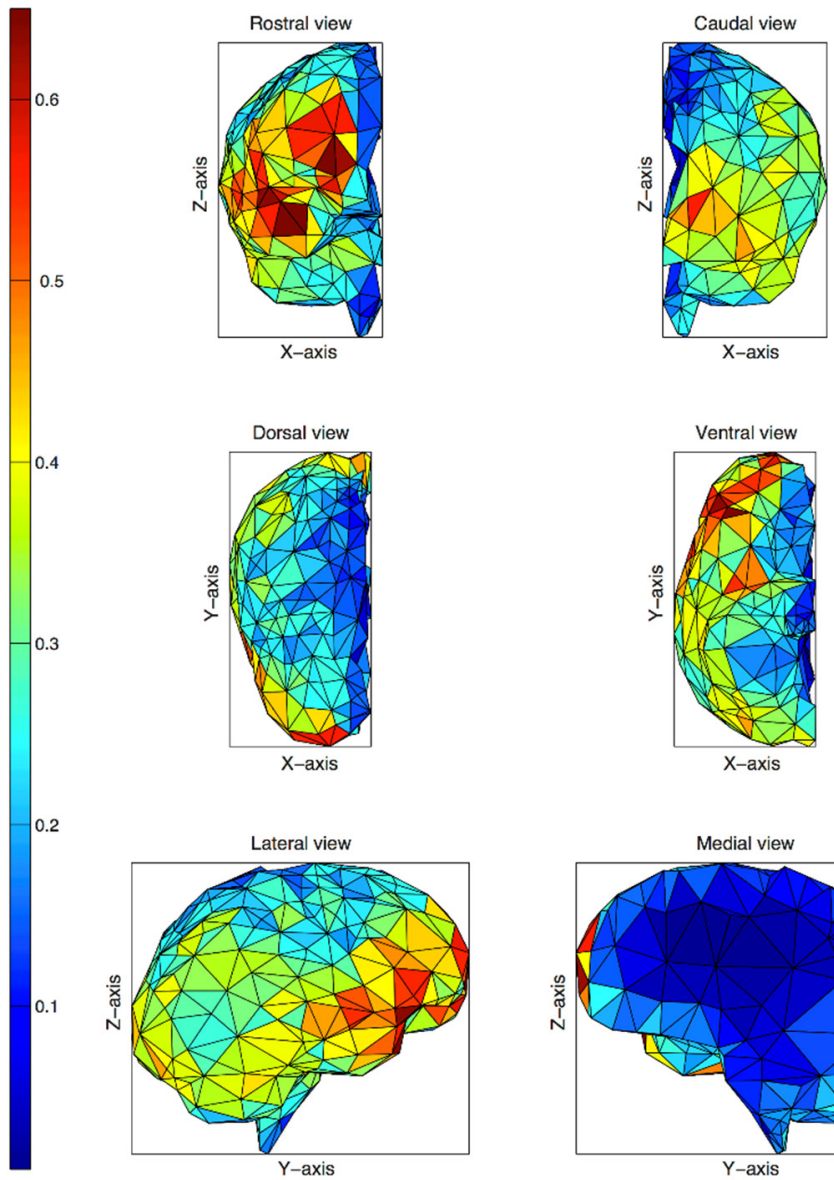

Figure 5: $\quad S A R$ distribution at $f=900 \mathrm{MHz}$. 
The obtained peak and average $S A R$ values for both polarization types and for at $f=900 \mathrm{MHz}$ and $f=1800 \mathrm{MHz}$ are given in Table 3 .

Table 3: $\quad$ Peak and average SAR values for different exposure scenarios.

\begin{tabular}{c|cc|cc}
\hline & \multicolumn{2}{|c}{ H } & \multicolumn{2}{c}{ V } \\
\hline \hline & $900 \mathrm{MHz}$ & $1800 \mathrm{MHz}$ & $900 \mathrm{MHz}$ & $1800 \mathrm{MHz}$ \\
\hline $\mathrm{SAR}_{\max }[\mathrm{W} / \mathrm{kg}]$ & 0,856486 & 4,390451 & 0,866016 & 2,678407 \\
\hline $\mathrm{SAR}_{\text {avg }}[\mathrm{W} / \mathrm{kg}]$ & 0,174457 & 0,411736 & 0,158206 & 0,348032 \\
\hline \hline
\end{tabular}

The calculated peak SAR values in the brain do not exceed the ICNIRP limits [6] for localized SAR (10 W/kg in the head and the trunk for the occupational exposure).

However, the exposure limit for the general public exposure limit $(2 \mathrm{~W} / \mathrm{kg}$ localized in the head and trunk) has been exceeded in the case of $1800 \mathrm{MHz}$ (both $1800 \mathrm{~V}$ and $1800 \mathrm{H}$ ).

\section{Conclusion}

The paper reviews the application of integral equation formulation in HF dosimetry featuring the human eye and brain, respectively, exposure to high frequency electromagnetic fields. The problems are solved by solving the governing equations using the hybrid BEM/FEM solution method for Helmholtz equation and MoM solution for surface integral equation. The governing equations are solved by means of certain BEM procedures. Illustrative computational examples are related to the assessment of SAR in the eye and brain due to the plane wave exposure.

Future work will be devoted to the extension of the present homogeneous brain computational model which would include different neighboring tissues.

\section{References}

[1] J.W. Hand: Modeling the interaction of electromagnetic fields $(10 \mathrm{MHz}-$ $10 \mathrm{GHz}$ ) with the human body: methods and applications, Physics in Medicine and Biology, vol. 53, no. 16, pp. 243-286, August 2008.

[2] D. Poljak, Electromagnetic fields: Environmental exposure. In: Nriagu JO (ed.) Encyclopedia of Environmental Health, volume 2, pp. 259-268 Burlington: Elsevier, 2011.

[3] D. Poljak, D. Cavka, H. Dodig, C. Peratta, A. Peratta, On the Use of Boundary Element Analysis in Bioelectromagnetics, Eng. Anal. Bound. Elem. (Special issue on Bioelectromagnetics) Vol. 49 (2014) pp. 2-14.

[4] N.F. Nielsen, J. Michelsen, J.A. Michalsen, and T. Schneider, "Numerical calculation of electrostatic field surrounding a human head in visual display environments", Journal of electrostatic, 36, pp. 209-223, 1996. 
[5] Krish. D. Singh, Nicola S. Longan and Bernard Gilmartin: "Three Dimensional Modeling of the Human Eye Based on Magnetic Resonance Imaging", Investigative Opthamology and Visual Science, 47, pp. 22722279, 2006.

[6] International Commission on Non-Ionizing Radiation Protection, "Guidelines for limiting exposure to time-varying electric, magnetic and electromagnetic fields (up to 300 GHZ)," Health Physics, vol. 74 No. 4, pp. 494-522, April 1998.

[7] International Commission on Non-Ionizing Radiation Protection, "Guidelines for limiting exposure to time-varying electric and magnetic fields (1 HZ-100 kHZ)," Health Physics, vol. 99 No. 6, pp. 818-836, December 2010.

[8] T. Nagaoka, K. Saito, M. Takahashi, K. Ito, S. Watanabe: Estimating specific absorption rates in pregnant women by using models at 12-, 20and 26-weeks' gestation for plane wave exposures, International Symposium on Electromagnetic Compatibility - EMC Europe, Hamburg, Germany, pp. 1-4, September 2008.

[9] T. Togashi, T. Nagaoka, K. Saito, M. Takahashi, K. Ito, S. Watanabe, T. Ueda, M. Saito, H. Ito, H. Osada: Development of Japanese 7-month pregnant woman model and evaluation of SAR generated by mobile radio terminals, $1^{\text {st }}$ European Conference on Antennas and Propagation EuCAP 2006, Nice, Italy, pp. 1-4, August 2006.

[10] M. Cvetković, D. Poljak, An Efficient Integral Equation Based Dosimetry Model of the Human Brain, Proc. of the 2014 International Symposium on Electromagnetic Compatibility (EMC Europe 2014), Gothenburg, Sweden, September 1-4, 2014, pp. 375-380.

[11] H. Dodig, D. Poljak, A. Peratta, Hybrid BEM/FEM Edge Element Computation of the Thermal Rise in The 3D Model of The Human Eye Induced By High Frequency EM Waves, 2012 International Conference on Software, Telecommunications and Computer Networks, Split, 2012.

[12] I. Laakso and A. Hirata, "Reducing the staircasing error in computational dosimetry of low-frequency electromagnetic fields," Phys. Med. Biol., 2012.

[13] D. Poljak, Human Exposure to Electromagnetic Fields, WIT Press, Southampton-Boston, 2003.

[14] D. Poljak, "Advanced Modeling in Computational Electromagnetic Compatibility”, New Jersey: John Wiley \& Sons, Inc., 2007.

[15] International Commission on Non-Ionizing Radiation Protection (ICNIRP) and International labour organization: "Visual display units: Radiation protection guidelines", International labour office, Geneva 1994.

[16] A. Hirata: Temperature increase in human eyes due to near-field and farfield exposures at $900 \mathrm{MHz}, 1.5 \mathrm{GHz}$, and 1.9 GHz, IEEE Transactions on Electromagnetic Compatibility, vol. 47, no. 1, pp. 68-76, February 2005.

[17] W. C. Chew, M. S. Tong, and B. Hu, Integral Equation Methods for Electromagnetic and Elastic Waves. Morgan \& Claypol Publishers, 2009. 
[18] R. Harrington, "Boundary integral formulations for homogeneous material bodies," Journal of electromagnetic waves and applications, vol. 3, no. 1, pp. 1-15, 1989.

[19] A. J. Poggio and E. K. Miller, "Integral equation solutions of threedimensional scattering problems," in Computer Techniques for Electromagnetics, Second Edition, R. Mittra, Ed. Hemisphere Publishing Corporation, 1987, ch. 4, pp. 159-264.

[20] K. Umashankar, A. Taflove, and S. Rao, "Electromagnetic scattering by arbitrary shaped three-dimensional homogeneous lossy dielectric objects," IEEE Transactions on Antennas and Propagation, vol. 34, no. 6, pp. 758$766,1986$. 